\title{
Experts' Understanding of Partial Derivatives Using the Partial Derivative Machine
}

\author{
David Roundy*, Eric Weber ${ }^{\dagger}$, Grant Sherer* and Corinne A. Manogue* \\ ${ }^{*}$ Department of Physics, Oregon State University, Corvallis, Oregon 97331 \\ ${ }^{\dagger}$ College of Education, Oregon State University, Corvallis, Oregon 97331
}

\begin{abstract}
We developed the Partial Derivative Machine (PDM) in response to difficulties we encountered in teaching students about mathematical concepts involving partial derivatives and total differentials that are needed in thermodynamics. The Partial Derivative Machine is a system that has four observable and controllable properties: two forces and two positions. However, of these four properties only two may be controlled independently. This context-dependence of independent and dependent variables enables the same sort of mathematical flexibility (and confusion) that is present in thermodynamics. Because the PDM is easy to use and understand, we hypothesized that it would allow us to explore the nature of experts' thinking about derivatives, even those unfamiliar with ideas in thermodynamics. In this paper, we present results from interviews with experts from several disciplines, as we explore how they understand partial derivatives when given an ambiguous prompt. The research question guiding this work is "How do experts think about partial derivatives?"
\end{abstract}

Keywords: partial derivative, thermodynamics, experiment

PACS: 01.40.Fk, 01.40.G, 05.70.-a

\section{INTRODUCTION}

Thermo is hard [1-4]. In a recent national workshop on the upper-division curriculum, approximately $1 / 3$ of the faculty indicated, in an informal show of hands, that they are uncomfortable enough with the content of thermodynamics that they would be reluctant to teach it.

There are various reasons why thermodynamics is hard. One reason is that thermodynamics involves a number of different quantities, e.g. entropy, temperature, pressure, volume. Of these four quantities, two are independent, but which two are controlled independently depends on the context. Partial derivatives represent important physical quantities, and this ambiguity in the independent variables makes it crucial to pay careful attention to which quantities are held fixed.

Thermodynamics is the first time that our students encounter scenarios in which the quantities held fixed when taking a partial derivative are ambiguous. In mathematics courses, students are taught that when taking partial derivatives, all the independent variables are held fixed, or at least all variables are able to be systematically varied and held constant. Nevertheless, through experience in the classroom we have found that most students come into our course with a firm belief that when taking a partial derivative everything else is held fixed.

We do not think that the issues we have observed with partial derivatives are limited to students. Indeed, we hypothesize that many of the issues we have observed are due to the ways in which different disciplines use and think about derivatives and partial derivatives. Moreover, by studying experts' thinking about partial derivatives, we will obtain a better benchmark for comparison in the study of students' thinking about those same ideas. In this study, we conducted small group interviews with experts in several STEM disciplines. These interviews are most similar to a clinical interview except that the group setting provides a means for participants to listen and respond to each others' ideas, rather than just the interviewer's. In the remainder of this paper, we will introduce the Partial Derivative Machine (PDM), and give the results of our analyses from the expert interviews.

What is a derivative? Students need to be fluent in looking at derivatives using multiple perspectives. We consider four different ways to understand and think about a derivative, each of which is useful in different scenarios.

1. A ratio of small changes. This is the limit definition of a derivative, and seems to be the first seen by students and the first forgotten.

2. A physical measurement to determine its value [5]. This involves measuring the small change in one quantity resulting from imposing a small change in another, and finding their ratio.

3 . The slope of the tangent to a curve.

4. The result of algebraic manipulation of a symbolic expression. For many students this is the primary understanding of "finding a derivative."

In the first two cases, it is most natural to think of a derivative as a number. One picks a point to take the derivative and finds a ratio as a number. While that number will be different at other points-making the deriva- 
tive actually a function - this aspect of the derivative may often be ignored. When considering the slope of the tangent to a curve, it is clearer that the derivative is a function, but it is also natural to think of the derivative as a number. When using the symbolic approach, the derivative is inherently a function, and while that function could be evaluated at a point, its value cannot be determined until its functional form is known.

What is a partial derivative? Partial derivatives differ from ordinary derivatives in important ways. How we understand this difference can vary with how we understand derivatives.

1,2 . When considering a ratio of small changes or a measurement, a partial derivative requires that we ask not only which quantities are changing, but also which quantities to hold fixed.

3. A tangent line turns to a tangent plane in two dimensions, and a partial derivative becomes the slope of the plane in a given direction at a given point.

4. The procedure to find a partial derivative of a symbolic expression is identical to that for an ordinary derivative, provided there are not interdependencies among the variables in the expression. It is unsurprising that many of our students believe that a partial derivative means "everything else is held fixed."

In thermal physics, the quantities that are being held fixed are context-dependent. How we respond to this ambiguity depends deeply on our concept of a derivative.

\section{THE PARTIAL DERIVATIVE MACHINE}

We have developed and used two versions of the Partial Derivative Machine (PDM). The first version of this device is documented in [6], and features a central system that is attached to four strings. The simplified version of this device - which will be discussed in this paper-is shown in Fig. 1, and consists of a fixed elastic system, which is constructed of springs and strings. In both versions, the elastic system may be manipulated using two strings independently. Each of these two strings has a scalar position that can be measured with a measuring tape and a tension that can be adjusted by adding to or removing weights from a hanger. Detailed instructions on constructing a Partial Derivative Machine, including a parts list and photographs of additional central systems, are available on our Paradigms website [7].

The usefulness of the PDM emerges because it is an exact mechanical analogue for a thermodynamic system. The system contains a potential energy $U$ (analogous to the internal energy) that cannot be directly measured. The system has four directly measurable-and controllable-state properties: two positions $x$ and $y$ and

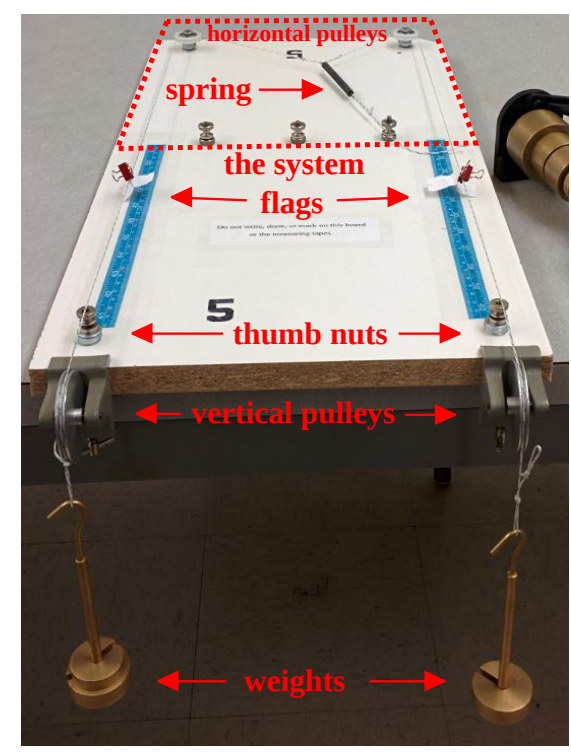

FIGURE 1. The Partial Derivative Machine.

two tensions $F_{x}$ and $F_{y}$. These four state properties play roles analogous to volume, entropy, pressure and temperature in a thermodynamic system.

As in thermodynamics, the choice of independent variables is context-dependent. While it is experimentally easiest to control the two weights as independent coordinates while measuring positions, it is sometimes theoretically more convenient to view the positions as the independent coordinates. Most notably, when using work to determine the potential energy, the positions are the "natural" variables, as seen in the total differential that is analogous to the thermodynamic identity:

$$
d U=F_{x} d x+F_{y} d y
$$

We can relate this total differential to the mathematical expression

$$
d U=\left(\frac{\partial F}{\partial x}\right)_{y} d x+\left(\frac{\partial F}{\partial y}\right)_{x} d y .
$$

By equating coefficients of $d x$ and $d y$, we can find expressions for the two forces as partial derivatives of the potential energy. This enables us to clarify the interdependence of the four directly observable quantities.

We use the PDM to teach a mathematical introduction to thermodynamics prior to our junior-level course in thermal physics, Energy and Entropy. This introduction uses seven contact hours, and covers the range of mathematical topics generally taught in undergraduate thermodynamics. We begin with total differentials and integration along a path, discuss partial derivatives and chain rules, mixed partial derivatives and Maxwell relations, and end with Legendre transformations. Throughout both this mathematical introduction and Energy and 
Entropy, a focus is placed on connecting the mathematical expressions with tangible reality [8].

\section{EXPERT INTERVIEWS}

To gain insight into our research question, we performed three expert interviews, each of which lasted around an hour. We began by introducing our experts to the PDM, and showing them how to manipulate the machine. Then we gave them the following prompt:

$$
\text { “Find } \frac{\partial x}{\partial F_{x}} \text {." }
$$

The purpose of the prompt was to understand their thinking about notation, their thinking about derivatives and partial derivatives, and how they related those ways of thinking to the PDM. During the interview the system was visible as in Fig. 1, which is in contrast to our classroom practice of hiding the system under a black box.

When we provided this prompt, we had not defined either $x$ or $F_{x}$, but rather let the interviewees discuss what these quantities might mean. After they had discussed the meanings of these terms, and we agreed that their meaning was sufficiently clear to us, we clarified if necessary that $x$ was the position of one flag (i.e. one string) and that $F_{x}$ was the tension in that string, which was determined by the weight.

An explicit goal of this task-in fact, a pedagogical design feature of the PDM-was to explore our experts understanding of partial derivatives outside the world of symbolic manipulation. We asked experts to "find" a partial derivative for which they are given no functional form, thus forcing them to move outside the wide array of partial derivative games involving manipulation of symbolic expressions [4].

During the course of the three interviews, a number of themes emerged. These themes were a combination of issues we noticed as we did the interviews and issues that emerged as we conducted the initial phases of data analysis. In the sections below we describe these themes and articulate how we saw each group of interviewees in the context of that theme.

We interviewed three groups of experts: physicists, engineers and mathematicians. For the physicists we interviewed one associate professor and one full professor. Both use computational methods in their research, which is in astrophysics and optics. We interviewed three engineers: one chemical engineer who is a full professor with considerable research and teaching expertise in thermodynamics, and two engineers who study student thinking and epistemology in engineering. Finally, we interviewed two mathematicians who are both assistant professors and whose research is in mathematics education at the collegiate level.

\section{Identifying $x$ and $F_{x}$}

Physicists The physicists' comments from the outset of the interview led us to believe that they had a similar understanding to ours. They noted that $x$ and $y$ were positions, and soon after concluded that $F_{x}$ was a tension on one string. However, they did spend some time thinking that the position $x$ might be an internal property of the system, noting that the position of one end of the spring was fixed in the system.

Engineers The engineers began the interview by almost immediately moving to measure the derivative using calculations and data collection. With some pressing, they revealed that they were measuring $F_{x}$ as tension. However, it was not until the middle of the interview that they chose to identify $x$ as the position of one particular string which led to them reevaluating their initial calculations for the derivative. Prior to this they expressed the two positions as $x_{L}$ and $x_{R}$.

Mathematicians From the outset of the interview, the two mathematicians were extremely puzzled by the subscript on $F_{x}$, noting that "we have not seen this type of notation before" and "it looks like a derivative but we are unsure what the symbols are." While they attended to the position of the strings, they did not interpret $F_{x}$ as the force related to $x$ until they were told this.

\section{What is a derivative?}

Physicists The physicists immediately (in under 2 minutes) jumped to measuring ratios of small changes, and also quickly checked that their changes were small enough by verifying that the relationship between the two quantities was linear. They never sought to determine a symbolic expression. that could describe the system, an issue that was important for other groups.

The physicists did articulate that the derivative itself is a function, but did not focus on trying to determine a functional form in order to differentiate it. Instead, they understood they were at best going to determine an approximation of the derivative and proceeded to focus on a ratio of small changes.

Engineers The engineers also immediately found a ratio of small changes and verified that the relationship between the quantities was linear. After some time, they determined they could create a single-variable plot of the points they collected and look at the tangent line to approximate the derivative. However, this was their only mention of using a graph in the entire interview.

Like the physicists, the engineers were also aware that the derivative was not a constant number, and their measurements which entailed varying different properties of the system in a systematic way support that the were focused on finding an approximation of the derivative. 
Mathematicians The mathematicians required 30 minutes and four prompts from the interviewer before finally recognizing that they could develop an experimental measurement for the derivative. They took data, and saw that larger changes in $x$ indicated a greater derivative, but did not construct a ratio from their measurements. They repeatedly returned to speculation regarding the functional form of $x$, even after being prompted to find a numeric value. In the process, one of the mathematicians asked the question, "What is the nature of what we are trying to find? Is it a number? a function?"

Eventually they recognized that since the derivative is the slope, a graph would allow them to measure a slope, and they decided to arrange their data in "ordered pairs" of $\left(F_{x}, x\right)$. This approach led them to determine an approach using slopes equivalent to a ratio of small changes, although they never did actually perform a division or write down a ratio.

\section{What about $y$ and $F_{y}$ ?}

Physicists One physicist decided that $F_{y}$ must be held fixed when measuring $\frac{\partial x}{\partial F_{x}}$, because we were taking a derivative with respect to $F_{x}$. Thus if we took the inverse derivative, $y$ would have been held fixed. He stated that "this is what he was taught." When he considered fixing the thumb nut, he stated that this (physical act?) would change $x$ to be a different function, a function of $F_{x}$ and $y$.

When asked how many independent variables there were, the physicists very quickly recognized that only two variables were independent, and soon acknowledged that you could do a change of variables so different pairs could be considered independent. Based on their gestures, we interpret that they used "physical" reasoning to decide they could fix either quantity. They expressed this in terms of $x=x\left(F_{x}, F_{y}\right)$ and $F_{y}=F_{y}\left(F_{x}, y\right)$.

Engineers Like the physicists, the engineers discussed holding either $y$ or $F_{y}$ fixed, and assumed at first the force needed to be held fixed. Late in the interview, one of the engineers asked the others how many independent variables they thought there were, and it was not immediately obvious that the others saw that there were only two independent variables. They wrote down a function $x$ with three arguments $x\left(F_{x}, F_{y}, y\right)$.

Mathematicians The mathematicians were very quick (once they knew what the variables meant) to recognize that $x$ depends on $F_{x}$ and $F_{y}$, and made effective use of the PDM to qualitatively investigate the dependence of the derivative $\frac{\partial x}{\partial F_{x}}$ on other quantities. They observed that holding $y$ fixed would give a different result than holding $F_{y}$ fixed well before making any quantitative measurements, but did not investigate this dependence any further. They simply assumed (like one physicist) that the other force was the "right" quantity to hold fixed.

\section{CONCLUSIONS}

Although we have not observed in physics students the mathematicians' specific confusion regarding $F_{x}$, the use of $x$ and $y$ as the two independent position variables-particularly in variables that are not spatially orthogonal-has consistently created confusion both in experts and novices. In future, we intend to name our four variables $x_{1}, x_{2}, F_{1}$, and $F_{2}$.

There is a spectrum of the ability of the experts to recognize and exploit the definition of a derivative as a ratio of small changes to answer the interview prompt. This definition was the most obvious to the physicists, but we note that the two physicists that we interviewed both had extensive computational experience, and this definition is a standard numerical method. We intend to interview additional physicists in order to obtain a more representative sample. Interestingly, in spite of the prominence of the limit definition of a derivative in mathematics courses and texts, our mathematicians took the longest to leverage this definition. This suggests to us that our students who will have received most of their training about derivatives from mathematicians may require scaffolding to learn to use this definition explicitly and thoughtfully in our courses.

All three groups of experts reached the conclusion that when differentiating with respect to $F_{x}, F_{y}$ was more natural to hold fixed than $y$. The prompt was deliberately ambiguous and there is no physical need for this assumption. We will think further about the pedagogical implication for this surprising result.

\section{ACKNOWLEDGMENTS}

The funding for this project was provided, in part, by the National Science Foundation under Grant Nos. DUE 0618877, DUE 0837829, DUE 1023120, and DUE 1323800.

\section{REFERENCES}

1. D. E. Meltzer, AIP Conf. Proc. 1179, 31-34 (2009).

2. W. Christensen, and J. Thompson, Proc. RUME (2010).

3. J. R. Thompson, C. A. Manogue, D. J. Roundy, and D. B. Mountcastle, AIP Conf. Proc 1413, 85-88 (2012).

4. M. B. Kustusch, D. Roundy, T. Dray, and C. A. Manogue, Phys. Rev. ST Phys. Educ. Res. 10, 010101 (2014).

5. D. Roundy, M. B. Kustusch, and C. Manogue, Am. J. Phys. 82, 39-46 (2014).

6. G. Sherer, M. B. Kustusch, C. A. Manogue, and D. Roundy, 2013 PERC Proceedings pp. 341-344 (2013).

7. physics.oregonstate.edu/portfolioswiki/courses:home:inhome.

8. C. A. Manogue, E. Gire, and D. Roundy, 2013 PERC Proceedings pp. 27-30 (2013). 